\title{
Convective heat transfer to a jet in cross-flow
}

\author{
by G. Cardone, C. Meola and G. M. Carlomagno
}

Università degli Studi di Napoli "Federico II" Dipartimento di Energetica, Termofluidodinamica Applicata e Condizionamenti Ambientali (D E T E C), P. Le Tecchio, 8080125 Napoli

\begin{abstract}
An experimental investigation is made to measure convective heat transfer coefficients over a plate due to the combined effects of a wind tunnel stream and a jet perpendicularly injected into the cross flow. Tests are carried out for varying the stream velocity and the velocity ratio of the jet to the stream. The turbulence level of the free stream is also varied by means of a turbulence promoter. An infrared scanning radiometer applied to the heated thin-foil technique is employed. Data is reduced in dimensionless form in terms of the Nusselt number $\mathrm{Nu}$, based on the nozzle exit diameter, or better the ratio $\mathrm{Nu} / \mathrm{Nu}_{0}$ where $\mathrm{Nu}_{0}$ is the Nusselt number relative to the undisturbed plate.
\end{abstract}

\section{Introduction}

Jets in cross-flow are encountered in many industrial applications including lifting jets for VISTOL aircraft during transition flight, turbine blade cooling and exhaust gas cooling, sewerage outfalls as well as jets of oil and gas entering the flow in oil wells. In this context, it is justified the wide interest both experimental and numerical of many researchers [1-7]. It is known that a jet discharging normal to a cross-flow results in a complex interaction between the two flows resulting in the deflection of the jet in the direction of the cross-flow. In particular, the cross-sectional area increases as the jet entrains fluid from the external stream and assumes a horseshoe shape with a pair of counter-rotating trailing vortices. Andreopolus and Rodi [1], by hot-wire measurements in the jet wake, found that for small ratios, $R$, of jet-to-crossflow velocity (i.e. $R=0.5$ ) the jet is bent over abruptly by the crossflow, while at higher $R$ values the jet penetrates further into the cross-stream and the bending over takes place more gradually; as a consequence, the extent of the wake region increases with increasing $R$. The lower pressure in the wake induces a lateral inward motion and also a downwash of the bent-over-jet; the inward motion transports high-momentum cross-stream fluid to the centreplane and, in the case of high $R$ values, leads to the formation of a wall-jettype layer with increased velocity, which displaces the wake region from the wall giving rise to unsteady vortices.

The flow field is characterised by a complex system of vortices: a horseshoe vortex system around the jet exit; ring-like vortices in the shear layer wall which become distorted with streamwise distance; the wake region beneath the downstream side of the jet contains streamwise wall vortices that lie above the flat wall and vertically oriented shedding vortices, the unsteady vortices observed by Andreopolus and Rodi [1] and defined by Kelso et al. [4] as "upright vortices". The latter bear similarity to the vortex street downstream of a solid cylinder.

Recently, Carlomagno et al. [5] carried out experiments, by means of Particle Tracking Velocimetry (PTV), in a water tunnel at low free stream velocity for $R$ ranging from 2 up to 10 . They analysed the near field of the jet and found skewness of the velocity trend in the direction of the jet axis.

It has to be pointed out that the main efforts of previous works have been focused on the comprehension of the flow field dynamics with little attention to the heat transfer problem, while a jet in cross-flow constitutes a fundamental aspect of cooling problems.

In this context, the work of Goldstein and Taylor [6] deserves attention; measurements of heat transfer in the case of a row of jets entering a cross-flow at an angle of $35^{\circ}$, are performed by means of the naphthalene sublimation technique for a free stream velocity of $15 \mathrm{~m} / \mathrm{s}$ with $R$ ranging from 0.2 up to 2 . The results in terms of $h / h_{0}$ (with $h_{0}$ the heat transfer 
coefficient for an undisturbed plate) contours and distributions along the longitudinal direction show a maximum positioned at 0.75 diameters downstream from the nozzle edge; the value of such maximum varies from 1.65 up to 4.0 for $R$ varying from 0.2 up to 2.0 .

The emphasis of the present study is on the measurement of the convective heat transfer coefficients for varying the free stream velocity and the velocity ratio in the case of a round jet perpendicularly injected into the stream; the influence of the stream turbulence level on the heat transfer coefficient is also investigated.

\section{Experimental apparatus and technique}

Experimental tests are performed in an open circuit wind tunnel having a $300 \times 400 \mathrm{~mm}^{2}$ test section which is $1.1 \mathrm{~m}$ long; one side wall is made of a printed circuit board insulated at the back and with a hole, drilled at $20 \mathrm{~cm}$ from the plate edge (i.e. the tunnel throat), for the jet injection. The surface viewed by the infrared camera is coated with a thin layer of opaque paint, which has emissivity coefficient $\varepsilon=0.97$ in the wavelength of interest. The access window to the test section for the infrared camera is simply made of bioriented polyetilene; calibration of the radiometer takes into account its presence.

The plate edge is well aligned to the tunnel wall to avoid induced turbulence. The air jet issues from a circular nozzle of exit diameter $D=13.7 \mathrm{~mm}$; the air, supplied by a compressor, goes through a pressure-regulating valve, a heat exchanger, a flow-meter then to a plenum chamber where the temperature is metered and so through the nozzle into the wind tunnel section. The test apparatus is schematically sketched in Fig.1.

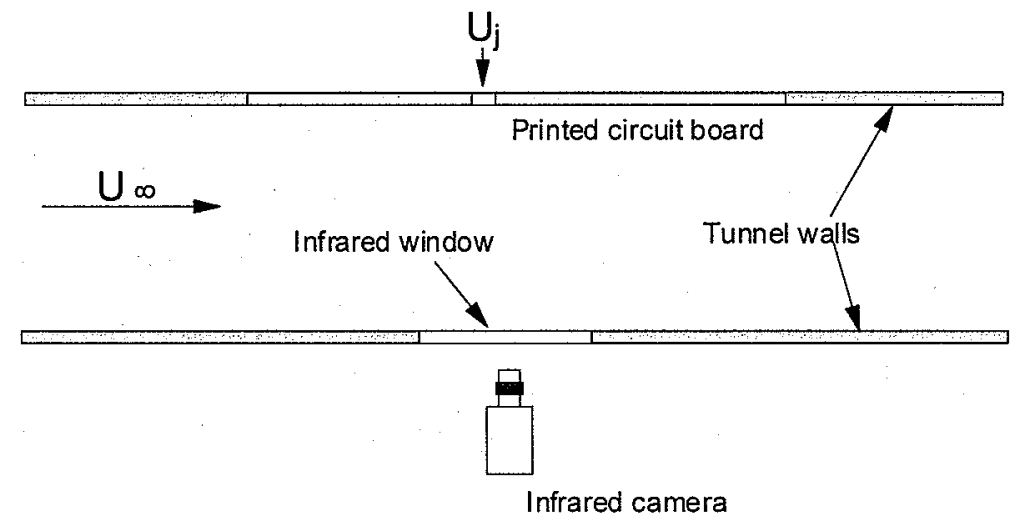

Fig.1. Experimental apparatus

Tests are carried out for varying the stream velocity $U_{\infty}$ from 5 up to $25 \mathrm{~m} / \mathrm{s}$ and the velocity ratio $R=U_{j} / U_{\infty}$ between 0 and 5 . Both laminar and turbulent flow configurations are analysed; turbulent conditions are attained by means of a turbulence promoter placed in the tunnel throat in correspondence of the conjunction to the plate edge. Measurements of boundary-layer thickness at X/D =-10 upstream of the jet exit are made by means of a hotwire anemometer for the free stream velocity $U_{\infty}=10 \mathrm{~m} / \mathrm{s}$; a value of the boundary-layer thickness $\delta$ of about $2 \mathrm{~mm}$ is found for the laminar case while $\delta=6 \mathrm{~mm}$ for the turbulent one. The turbulence level at that velocity (in the proximity of the wall) is about $0.5 \%$ and increases to about $10 \%$ when the promoter turbulence is inserted. Particular care is taken to maintain, during tests, the plenum jet temperature equal to the ambient one which coincides with the stream temperature.

The employed infrared thermographic system is the Agema Thermovision 900LW. The infrared camera measures the temperature map of the model wall which is correlated to the heat transfer coefficient by means of the steady state heated thin-foil technique [8]. The foil, 
as previously said, is a printed circuit board made of electrical conducting copper tracks arranged in a greek fret mode; after drilling, the electrical continuity for Joule heating is assured by welding points over the copper tracks $(4.5 \mathrm{~mm}$ wide) near the hole edge.

The convective heat transfer coefficient $h$ is calculated from the relationship:

$$
h=\frac{\dot{q}-\dot{q}_{1}}{T_{w}-T_{a w}}
$$

where $\dot{q}$ is the Joule heating, $\dot{q}_{l}$ includes losses due to radiation, $\dot{q}_{r}$, and lateral conduction, $\dot{q}_{G}, T_{w}$ and $T_{\text {aw }}$ are the wall and the adiabatic wall temperatures respectively.

The radiative thermal losses $\dot{q}$ may be evaluated according to the Stefan-Boltzman law:

$$
\dot{q}_{r}=\varepsilon \sigma\left(T_{w}^{4}-T_{a}^{4}\right)
$$

where $\varepsilon$ is the total emissivity coefficient, $\sigma$ is the Stefan-Boltzman constant and $T_{a}=T_{a w}$.

The tangential conduction $\dot{q}_{c}$ may be evaluated by means of the second derivative of the wall temperature $T_{w}$ :

$$
\dot{q}_{c}=s\left(\lambda_{p} \frac{\partial^{2} T_{w}}{\partial x^{2}}+\lambda_{n} \frac{\partial^{2} T_{w}}{\partial y^{2}}\right)
$$

where $s$ is the thickness of the foil, $\lambda_{p}$ and $\lambda_{n}$ the thermal conductivity coefficient along the tracks and perpendicular to them, respectively.

\section{Results}

Thermograms of wall temperatures $T_{w}$ for $U_{\infty}=10 \mathrm{~m} / \mathrm{s}$ and $R=1$ and 5 are shown in Fig.2 a) and b) respectively, the thermogram reported in Fig.2c is instead relative to $U_{\infty}=$ $10 \mathrm{~m} / \mathrm{s}$ and $R=5$ for the case with the turbulence promoter upstream.

In the laminar case, outside of the wake induced by the jet, it is clearly observable the development of the boundary layer over the plate with increasing wall temperature in the downstream direction; in addition, as $R$ increases the isotherms tends to wrap around the nozzle exit and to diverge downstream. As the turbulence level of the free stream increases (Fig.2C) the jet is prevented to enlarge over the plate and so it covers a surface zone smaller than that in the case of laminar flow conditions (Fig.2b).

Data is reduced in dimensionless form in terms of the Nusselt number $\mathrm{Nu}$ based on the jet exit diameter, $D$ :

$$
N u=\frac{h D}{\lambda}
$$

where $h$ is computed by Eq. $1, \lambda$ is the thermal conductivity of air evaluated at film temperature.

In particular, $\mathrm{Nu}$ is normalised with respect to $N u_{0}$, i.e. the Nusselt number relative to the undisturbed plate without jet injection and with the hole closed to simulate the case of a flat plate. The streamwise distribution of $N u_{0}$ for several values of the free stream velocity $U_{\infty}$ is reported in Fig.3. The Nusselt number, apart from in the initial zone close to the hole edge which is disturbed by the presence of the welding over the copper tracks, is constant in the streamwise direction $x / D$ and increases with $U_{\infty}$ The presence of the turbulence promoter entails an abrupt increase of $N U_{0}$ as can be seen by comparing the two profiles relative to $U_{\infty}$ $=10 \mathrm{~m} / \mathrm{s}$ with and without turbulence promoter. 


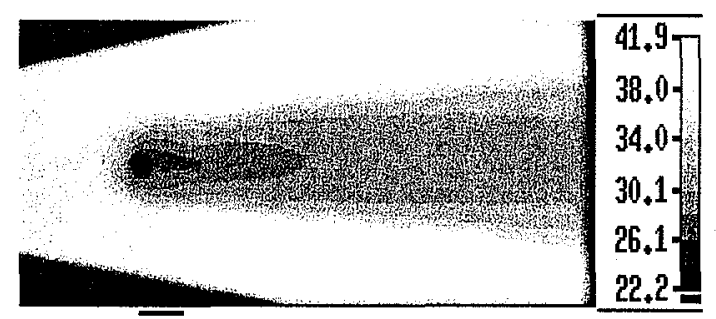

a) $U_{\infty}=10 \mathrm{~m} / \mathrm{s}, R=1$

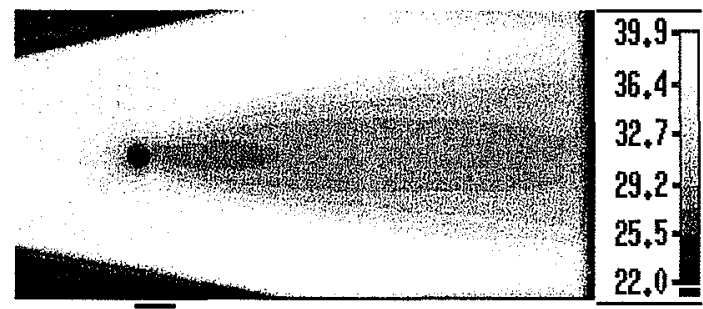

b) $U_{\infty}=10 \mathrm{~m} / \mathrm{s}, R=5$

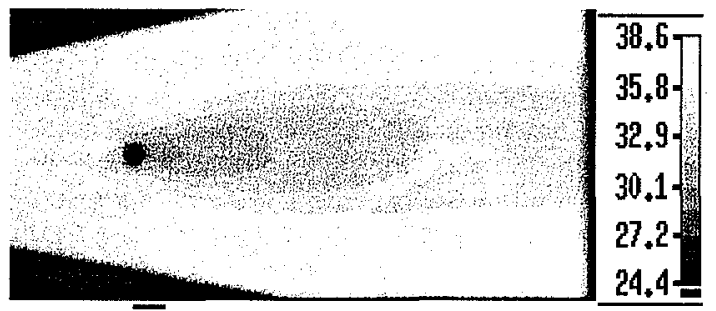

c) $U_{\infty}=10 \mathrm{~m} / \mathrm{s}, R=5$ with turbulence promoter

Fig. 2. $T_{w}$ distribution

Results are presented as $\mathrm{Nu} / \mathrm{Nu}_{0}$ maps in Fig.4 for several test conditions; a circle denotes the position of the jet exit. In general, $\mathrm{Nu} / \mathrm{Nu}$ o takes values equal to unity outside the jet wake where heat transfer is due only to the stream; immediately downstream of the injection hole a low heat transfer zone is present; further downstream the Nusselt number increases and attains its maximum value at about $x / D=1.75$ starting from the nozzle centre. This is in general agreement with results presented by Goldstein and Taylor [6] even if the latter authors considered jets inclined at $35^{\circ}$ to the surface. By comparing data relative to the different values of the stream velocity and the velocity ratio, also those not shown here, the position of this maximum seems to be independent of the flow conditions $U_{\infty}$ and $R$.

As far as the quantitative value of the $\mathrm{Nu} / \mathrm{Nu}$ o peak is concerned, two different cases have to be considered: laminar and turbulent flow conditions. For laminar flow conditions, the peak increases as $U_{\infty}$ increases; in fact, it attains values of about 5.6 for $U_{\infty}=5 \mathrm{~m} / \mathrm{s}$ and 6.5 for $U_{\infty}=10 \mathrm{~m} / \mathrm{s}$. As transition to turbulent flow occurs, $N u_{\circ}$ increases abruptly as can be seen by comparing the $N u_{0}$ profiles relative to the different values of the stream velocity and in particular those relative to $U_{\infty}=10 \mathrm{~m} / \mathrm{s}$ with and without turbulence promoter of Fig.3. It is worth noting that for the employed wind tunnel and the fixed location over the plate surface, transition to turbulent flow occurs at about $U_{\infty}=18 \mathrm{~m} / \mathrm{s}$. As a consequence $\mathrm{Nu} / \mathrm{Nu} u_{0}$ decreases since the heat transfer contribution of the jet is unchanged. This can explain the smaller heat transfer values found by Goldstein and Taylor [6] for a velocity stream of $15 \mathrm{~m} / \mathrm{s}$ and a Reynolds number of 11.000 against 8.700 relative to $U_{\infty}=10 \mathrm{~m} / \mathrm{s}$ for present data. 


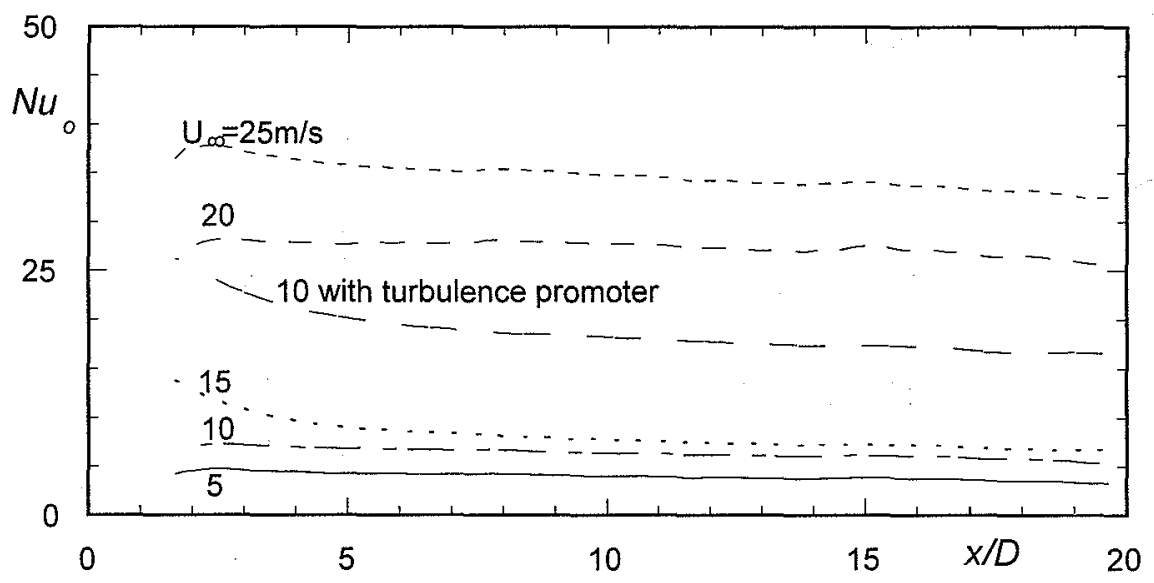

Fig. 3. Streamwise Nusselt number distribution over the undisturbed plate

Maps relative to $U_{\infty}=5 \mathrm{~m} / \mathrm{s}$ (Fig. $4 \mathrm{a}-\mathrm{c}$ ) show a secondary peak, less pronounced than the first one, at about 3.25 diameters.

According to literature, the minimum, which is located about $1 D$ downstream, may be ascribed to the stagnation region created by the injection. The presence of maximum heat transfer regions downstream may be associated with the formation of eddies in the wake. In fact, as the jet separates from the wall, for increasing $R$, the mainstream flow penetrates beneath the jet giving rise to the formation of the wake vortices which entail high local heat transfer. These vortices are unsteady and may be fast swept away as the velocity, and/or the turbulence level, of the free stream increases, as a consequence, the heat transfer peaks become much milder or vanish (Fig.4d-e).

The two lateral $\mathrm{Nu} / \mathrm{Nu} u_{o}$ minima at about $1 D$ should correspond to the position of the two trailing votices which accompany the jet issuing from the nozzle.

It has to be pointed out that, by enhancing the spatial resolution (infrared camera at the minimum lens focus distance or by using extension rings) it should also be possible to visualize the horseshoe vortices close to the nozzle exit; however, the presence of the welding points over the copper tracks disturb the $T_{w}$ distribution close to the hole contour and do not allow for a correct interpretation of results there. So that, a new ad hoc circuit, without any welding point, has been designed and new tests are underway.

\section{Conclusions}

An infrared scanning radiometer applied to the heated thin-foil technique has been employed to measure convective heat transfer coefficients over a plate subjected to the combined effects of a wind tunnel stream and a jet perpendicularly injected into the crossflow. Data has been reduced in non-dimensional form in terms of the Nusselt number normalised with respect to the Nusselt number relative to the undisturbed plate. Apart from the region very close to the nozzle exit where the presence of the welding points over copper tracks disturb the wall temperature distribution and do not allow for reliable data, results are in general agreement with literature. Infrared thermography appears able to detect the vortex structures associated with the complex dynamics of a jet in a cross-flow. 
http://dx.doi.org/10.21611/qirt.1998.044

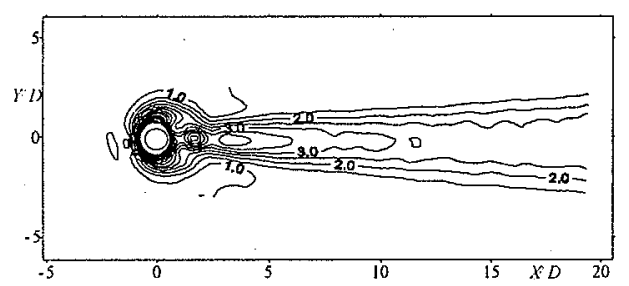

a) $U_{\infty}=5 \mathrm{~m} / \mathrm{s}, R=1$

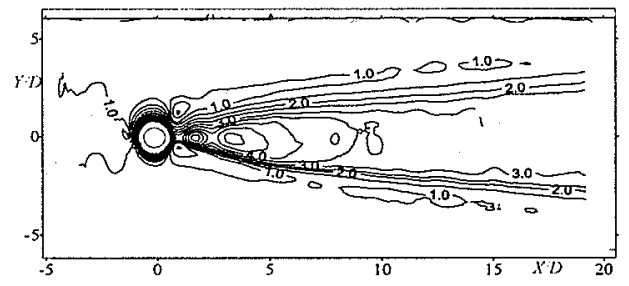

b) $U_{\infty}=5 \mathrm{~m} / \mathrm{s}, R=3$

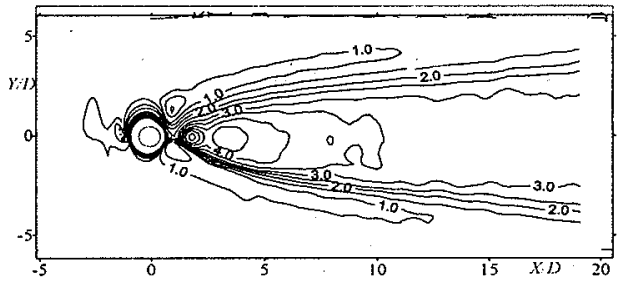

c) $U_{\infty}=5 \mathrm{~m} / \mathrm{s}, R=5$

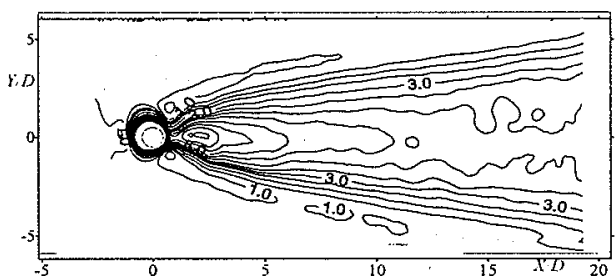

d) $U_{\infty}=10 \mathrm{~m} / \mathrm{s}, R=5$

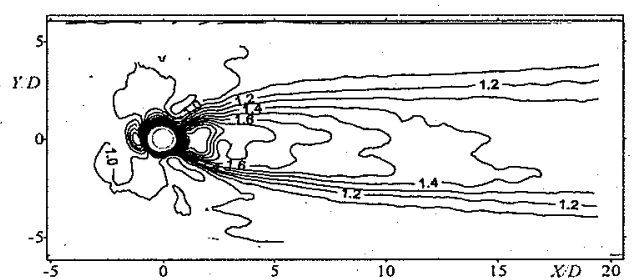

e) $U_{\infty}=10 \mathrm{~m} / \mathrm{s}, R=5$, with turbulence promoter

Fig. 4. Maps of $\mathrm{Nu} / \mathrm{Nu}$

\section{REFERENCES}

[1] ANDREOPOULOS (J.) and RODI (W.) - Experimental investigation of jets in a crossflow, J. Fluid Mech. Vol. 138, pp. 93-127, 1984.

[2] SYKES (R. I.), LEWELLEN (W. S.) and PARKER (S. F.) - On the vorticity dynamics of a turbulent jet in a crossflow. J. Fluid Mech. Vol. 168, pp. 393-413, 1986.

[3] COELHO (S. L. V.) and HUNT (J. C. R.) - The dynamics of the near field of strong jets in crossflows, J. Fluid Mech. Vol. 200, pp. 95-120, 1989.

[4] KELSO (R. M.), LIM (T. T.) and PERRY (A. E.) - An experimental study of round jets in cross-flow, J. Fluid Mech. Vol. 306, pp. 111-144, 1996.

[5] CARLOMAGNO (G. M.), CENEDESE (A.) and de ANGELIS (G.) - PTV analysis of jets in cross-flow, Proceedings of $9^{\text {th }}$ Intern. Symp. on Applications of Laser Techniques to Fluid Mechanics, vol.2, 38.3 1-38.3 7, Lisbon, 1998.

[6] GOLDSTEIN (R. J.) and TAYLOR (J. R.) - Mass transfer in the neghborhood of jets entering a crossflow, J. Heat Transfer vol.104, pp. 715-721; 1982.

[7] SCHERER (V), WITTIG (S.) BITTLINGER (G.) and PFEIFFER (A.) - Thermographic heat transfer measurements in separated flows, Experiments in fluids vol. 14, pp. 17-24, 1993.

[8] CARLOMAGNO (G. M.) and de LUCA (L.) - Infrared thermography in heat transfer, in Handbook of Flow Visualization,. Yang W.J. ed. chap. 32, pp. 531-553, 1989. 